\title{
Digit symbol coding and general cognitive ability in schizophrenia: worth another look?
}

\author{
Dwight Dickinson
}

\begin{abstract}
Summary
For decades, schizophrenia researchers have sought to map specific aspects of cognitive performance onto specific neurobiological systems in hopes of dividing broad cognition and neurobiology into more tractable components. Recent findings from studies using neuropsychological test batteries, in combination with
\end{abstract}

emerging neurobiological evidence, argue for a complementary focus on more generalised cognitive and biological dimensions.

\section{Declaration of interest}

None.
Dwight Dickinson (pictured) is Associate Professor of Psychiatry with the University of Maryland School of Medicine and VISN 5 Mental Illness Research, Education and Clinical Center.

An article of faith in schizophrenia cognitive research is that it should be possible to link relatively specific behavioural processes (e.g. executive functioning performance as measured by card sorting tasks) to relatively specific neurobiological substrates (e.g. activity in dorsolateral prefrontal cortex systems), and that such work will carve schizophrenia cognition into more discrete, genetically simpler, and potentially treatable components. In the face of complex neurobiology and significant illness heterogeneity, progress along this path has been slow. Advances in genetics and new electrophysiological and neuroimaging technologies promise better defined and ever more specific targets on the biological side of the analysis. At the same time, there are active efforts to translate precise experimental paradigms into user-friendly instruments for the clinical measurement of behaviour. ${ }^{1}$ It seems likely that these advances will yield reliable and specific brain/cognition associations, some of which may be particular to subgroups within the schizophrenia spectrum. But will the associations prove as useful in deciphering schizophrenia as we hope? On the behavioural side, in particular, the price of progress seems to be a focus on increasingly rarefied behaviours. ${ }^{2,3}$ Yet, it is not clear that cognitive functioning can be parsed meaningfully to the same degree as underlying biology. It may be that, in the drive to understand the illness at a molecular and mechanistic level, the field will lose its focus on the complex systems of behaviour that are ultimately the heart of the illness and the keys to functioning in the community. Notwithstanding waves of exciting new work, it remains the case that schizophrenia impairs most complex cognitive operations to a similar degree with few exceptions, that the largest documented effect sizes in the schizophrenia cognitive literature have been for traditional and often simple neuropsychological measures, and that this is the class of measures that is most clearly associated with functional outcome among people with schizophrenia (reviewed by Heinrichs ${ }^{4}$ ). These straightforward and persistent findings should raise questions among the faithful.

\section{Digit symbol coding in schizophrenia}

Neuropsychological measures have been central to schizophrenia cognitive research for decades, and their wide use continues. ${ }^{5}$ Familiar examples include word list learning (i.e. verbal memory), card sorting (i.e. executive functioning), and span tasks (i.e. working memory). In contrast to these measures, less attention has been devoted to coding tasks, such as the Wechsler Digit Symbol Substitution Test. ${ }^{6}$ These tasks - which have not changed fundamentally since their introduction a century ago ${ }^{7}-$ reflect the coordination and speeded performance of a number of uncomplicated scanning, matching and motor operations. Performance does not appear to be associated with particular regional or functional brain systems. However, the measures are extremely brief ( $5 \mathrm{~min}$ to administer and score), are more reliable than other well-known neuropsychological measures, are sensitive to a wide variety of developmental and clinical conditions, as well as normal ageing, and are often present in research batteries.

Surprisingly, a recent meta-analysis demonstrated that these simple measures discriminate people with schizophrenia from comparison individuals better than the more widely studied neuropsychological instruments. ${ }^{8}$ Across 37 studies, 1961 people with schizophrenia and 1444 healthy controls, coding tasks showed a schizophrenia impairment that was both substantial $(g=-1.57)$ and significantly larger than effects derived for other measures. Indeed, only one of 36 comparison measures considered in the meta-analysis showed a nearly comparable effect across studies (category fluency, $g=-1.45$ ). The coding task effect was reasonably homogeneous despite striking differences in samples and designs from study to study (range $g=-1.05$ to -2.02 ). The magnitude of the effect was not sensitive to medication status or symptomatology. The coding task effect did differ in predictable ways as a function of schizophrenia chronicity/severity groupings (i.e. first episode $<$ chronic $<$ very-early onset). Still, these differences were modest, and in each of these patient subgroups the coding task effect size was larger than effects obtained for other cognitive variables. In addition, an offshoot analysis revealed a substantial coding task effect size among unaffected relatives of people with schizophrenia. Other studies have reported that coding performance also predicts functional status. Overall, coding tasks produce a robust impairment signal that differentiates healthy controls from people with schizophrenia and their close relatives better than other measures, and indexes poor prognosis and functional disability.

The meta-analysis also highlighted the generalised nature of cognitive impairment in schizophrenia (see also Heinrich \& Zakzanis $^{9}$ ). Across all measures, the mean weighted effect size was $g=-0.98$, and a number of familiar measures, including IQ, word list learning, story memory, mental arithmetic, and the 'AX' version of the continuous performance test, showed values well above this average (range $g=-1.10$ to -1.29 ). Thus, the 
review reaffirmed a broadly generalised cognitive deficit in schizophrenia, and provided evidence of a small, disproportionate effect on simple coding tasks and, to a lesser degree, on some other measures.

\section{General cognitive ability in schizophrenia}

Other work with collaborator Jim Gold suggests a 'general cognitive ability' framework for understanding these results. In schizophrenia investigations, generalised cognitive impairment has often been treated as a nuisance variable or artefact that obscures more cognitive domain-specific effects (e.g. in domains of verbal memory or executive functioning; see Albus \& Hubmann ${ }^{10}$ ). However, the general ability findings are robust and similar across heterogeneous schizophrenia subgroups and very different sorts of analyses, suggesting that they should not be dismissed as artefact. We have used statistical modelling to characterise the structure of cognitive performance within the schizophrenia group, ${ }^{11}$ and of the cognitive deficit in affected persons relative to healthy controls. ${ }^{12,13}$ The latter studies were consistent in suggesting that about two-thirds of the diagnosis effect on cognitive performance was mediated through a general ability factor, with a limited number of small effects (notably, in the processing speed domain) $)^{12,13}$ (Fig. 1).

More generally, the factor-analytic literature argues for a hierarchical model of cognitive test performance - with individual tests loading on first-order cognitive domain factors, and these factors loading in turn on a single, higher-order general cognitive ability factor. The heart of the hierarchical model is the idea that generalised cognitive ability or ' $g$ ' underlies much, but not all, of performance in different cognitive domains and on individual cognitive measures. ${ }^{14}$

Thus, our modelling work and meta-analytic results converged on key points: first, highlighting the prominence of the generalised cognitive impairment in schizophrenia; and second, showing that

(a)

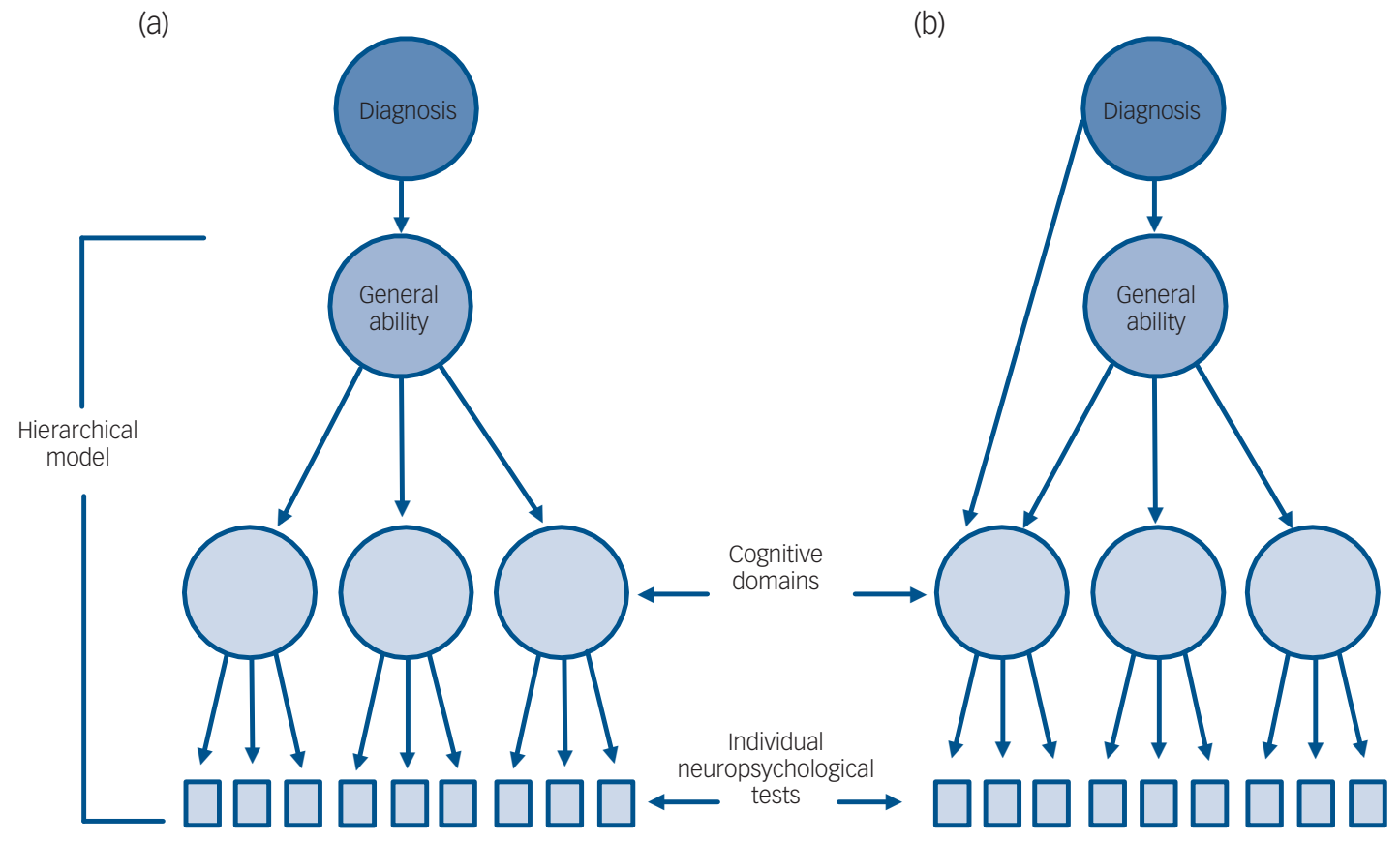

Fig.1 Diagnosis effect on cognitive performance. Between-groups cognitive structure can be modelled by adding diagnosis as a grouping factor, and estimating parameters from diagnosis through the general cognitive factor to individual performance domains and, at the same time, from diagnosis directly to the domain factors. (a) Illustrates diagnosis effects entirely mediated through a higher order general cognitive ability factor. (b) Illustrates a model in which diagnosis affects performance domains through the general ability factor and has direct effects certain measures and domains, including coding tasks, were disproportionately impaired to a small but significant degree. It seems to follow from the findings regarding generalised cognitive structure in schizophrenia that much of what is measured by traditional neuropsychological assessment measures - whether they are generally held to tap verbal memory or executive functioning or some other domain - is broad cognitive ability, not independent, domain-specific performance.

\section{Significance of findings from neuropsychological battery studies}

These lines of research frame a critical question: do general cognitive ability interpretations of neuropsychological findings in schizophrenia mainly reflect limitations of existing measures or do they reveal something fundamental about the structure of cognition in this disorder? One possibility is that coding and most other neuropsychological tasks are so inherently multifactorial that their utility for probing specific biological or treatment effects in schizophrenia is sharply limited. ${ }^{1}$ If so, then measurement techniques adapted from cognitive neuroscience, serving as intermediate phenotypes for genetically defined targets, may finally enable researchers to carve schizophrenia cognition 'at its joints., ${ }^{2,3}$ However, this outcome is uncertain. Even assuming further progress in isolating discrete behaviours in schizophrenia and connecting them with biological markers, it could turn out that the target behaviours have been stripped down to a degree that leaves their relationships to important everyday outcomes obscure.

Another possibility is that the generalised cognitive deficit is a fundamental manifestation of schizophrenia. If so, then one path to improved understanding of the pathophysiology of cognitive impairment may emerge from a fresh look at the neurobiological associations of general ability. Several lines of evidence emerging 
in the field are consistent with the hypothesis that the neurobiology underlying generalised cognitive dysfunction is likewise general in nature. Examples include: (a) broadly reduced grey matter and neuronal arborisation; (b) diminished myelin density and fibre coherence in major white matter tracts; (c) poor signal integration at the level of the cell and the network due to cortical background noise and reduced neural synchrony; and (d) abnormalities associated with the brain's main excitatory and inhibitory neurotransmitters, glutamate and $\gamma$-amniobutyric acid. ${ }^{12}$ Importantly, related findings are emerging in healthy groups and among unaffected relatives of people with schizophrenia. ${ }^{12}$ Some have hypothesised roles for even more general biological processes, such as oxidative stress, inflammation and energy metabolism, ${ }^{15}$ possibilities that might begin to recast schizophrenia as a general, systemic disorder, rather than a focal brain disease. Findings from outside the schizophrenia literature supporting 'generalist genes' for cognitive development, performance and impairment also seem relevant. ${ }^{16}$

Neuropsychological measures show an excellent ability to discriminate affected individuals and their relatives from controls and to index disability among those affected. ${ }^{4}$ This may be precisely because they are sensitive to such generalised biological effects. Coding tasks, in particular, appear to measure impairment in integrating or coordinating distributed brain networks, more so than failures connected to specific sub-processes. This 'systems' level is a potentially critical level of analysis for schizophrenia and, therefore, the relatively diffuse measurement focus of coding tasks and other similar measures might be seen as a strength of the instruments rather than a weakness. ${ }^{1}$ In sum, emerging evidence provides a basis for renewed interest in the generalised cognitive deficit in schizophrenia and suggests that investigation of associations at more integrated levels of cognition and neurobiology should be pursued in balance with more mechanistically targeted research.

Dwight Dickinson, PhD, University of Maryland School of Medicine, VISN 5 Mental Illness Research, Education and Clinical Center, Baltimore, Maryland, USA. Email: Dwight.Dickinson@va.gov

First received 4 Jan 2008, final revision 18 Jun 2008, accepted 24 Jun 2008

\section{References}

1 Carter CS, Barch DM. Cognitive neuroscience-based approaches to measuring and improving treatment effects on cognition in schizophrenia: the CNTRICS initiative. Schizophr Bull 2007; 33: 1131-7.

2 MacDonald AW 3rd, Carter CS, Flory JD, Ferrell RE, Manuck SB. COMT Val158Met and executive control: a test of the benefit of specific deficits to translational research. J Abnorm Psychol 2007; 116 306-12.

3 Tan HY, Chen Q, Goldberg TE, Mattay VS, Meyer-Lindenberg A, Weinberger DR, Callicott JH. Catechol-O-methyltransferase Val158Met modulation of prefrontal-parietal-striatal brain systems during arithmetic and temporal transformations in working memory. J Neurosci 2007; 27: 13393-401.

4 Heinrichs RW. The primacy of cognition in schizophrenia. Am Psychol 2005 60: $229-42$.

5 Nuechterlein KH, Green MF, Kern RS, Baade LE, Barch DM, Cohen JD, Essock S, Fenton WS, Frese FJ 3rd, Gold JM, Goldberg T, Heaton RK, Keefe RSE, Kraemer $\mathrm{H}$, Mesholam-Gately R, Seidman LJ, Stover E, Weinberger DR, Young AS, Zalcman S, Marder SR. The MATRICS Consensus Cognitive Battery, part 1: test selection, reliability, and validity. Am J Psychiatry 2008; 165: 203-13.

6 Wechsler D. Wechsler Adult Intelligence Scale (3rd edn). Psychological Corporation, 1997.

7 Boake C. From the Binet-Simon to the Wechsler-Bellevue: tracing the history of intelligence testing. J Clin Exp Neuropsychol 2002; 24: 383-405.

8 Dickinson D, Ramsey M, Gold JM. Overlooking the obvious: a meta-analytic comparison of digit symbol coding tasks and other cognitive measures in schizophrenia. Arch Gen Psychiatry 2007; 64: 532-42.

9 Heinrichs RW, Zakzanis KK. Neurocognitive deficit in schizophrenia: a quantitative review of the evidence. Neuropsychology 1998; 12: 426-45.

10 Albus M, Hubmann W. Neuropsychological functions in patients with schizophrenia. Am J Psychiatry 1999; 156: 1665-6.

11 Dickinson D, Ragland JD, Calkins ME, Gold JM, Gur RC. A comparison of cognitive structure in schizophrenia patients and healthy controls using confirmatory factor analysis. Schizophr Res 2006; 85: 20-9.

12 Dickinson D, Ragland JD, Gold JM, Gur RC. General and specific cognitive deficits in schizophrenia: Goliath defeats David? Biol Psychiatry 2008; in press.

13 Dickinson D, Iannone VN, Wilk CM, Gold JM. General and specific cognitive deficits in schizophrenia. Biol Psychiatry 2004; 55: 826-33.

14 Carroll JB. Human Cognitive Abilities: A Survey of Factor-Analytic Studies. Cambridge University Press, 1993

15 Hanson DR, Gottesman, II. Theories of schizophrenia: a genetic-inflammatoryvascular synthesis. BMC Med Genet 2005; 6: 7.

16 Plomin R, Kovas Y. Generalist genes and learning disabilities. Psychol Bull 2005: 131: 592-617. 